\title{
Investigation of Asymmetric Thrust Detection with Demonstration in a Real-Time Simulation Testbed
}

\author{
Amy Chicatelli ${ }^{\dagger}$, Aidan W. Rinehart ${ }^{\ddagger}$ and T. Shane Sowers ${ }^{\S}$ \\ Vantage Partners LLC, Brook Park, OH 44142 \\ Donald L. Simon** \\ NASA Glenn Research Center, Cleveland, OH 44135
}

\begin{abstract}
The purpose of this effort is to develop, demonstrate, and evaluate three asymmetric thrust detection approaches to aid in the reduction of asymmetric thrust-induced aviation accidents. This paper presents the results from that effort and their evaluation in simulation studies, including those from a real-time flight simulation testbed. Asymmetric thrust is recognized as a contributing factor in several Propulsion System Malfunction plus Inappropriate Crew Response (PSM+ICR) aviation accidents. As an improvement over the state-of-the-art, providing annunciation of asymmetric thrust to alert the crew may hold safety benefits. For this, the reliable detection and confirmation of asymmetric thrust conditions is required. For this work, three asymmetric thrust detection methods are presented along with their results obtained through simulation studies. Representative asymmetric thrust conditions are modeled in simulation based on failure scenarios similar to those reported in aviation incident and accident descriptions. These simulated asymmetric thrust scenarios, combined with actual aircraft operational flight data, are then used to conduct a sensitivity study regarding the detection capabilities of the three methods. Additional evaluation results are presented based on pilot-in-the-loop simulation studies conducted in the NASA Glenn Research Center (GRC) flight simulation testbed. Data obtained from this flight simulation facility are used to further evaluate the effectiveness and accuracy of the asymmetric thrust detection approaches. Generally, the asymmetric thrust conditions are correctly detected and confirmed.
\end{abstract}

\section{Introduction}

A symmetric thrust is cited as the cause of several loss of control aviation incidents and accidents. ${ }^{1,2,3}$ As noted in the propulsion system malfunction plus inappropriate crew response (PSM+ICR) reports, the detection of unintended asymmetric thrust conditions needs to be performed in a timely manner in order for there to be sufficient time for recovery. This effort was part of a feasibility study that evaluated three asymmetric thrust detection methods. This paper presents the results from that study which was conducted in simulation to demonstrate that asymmetric thrust conditions can be detected. In addition, to test effectively any proposed asymmetric thrust detection method, a realistic flight simulation environment is necessary. The NASA GRC flight simulation laboratory provides an opportunity to evaluate the effectiveness of the proposed methods with a pilot-in-the-loop. However, additional work would be necessary to address whether this information should be annunciated to the flight crew

The remainder of the paper is organized as follows. The paper begins with a section that provides the motivation for this work and describes the significance and improvement over state of the art. Following that, the description of three developed asymmetric thrust detection methods is provided. Next, results from a simulation-based sensitivity study and the application of these methods in a real-time flight simulation laboratory are presented. Finally, a summary that outlines the effectiveness of the asymmetric thrust detection approaches is presented along with generalized concluding remarks about the research effort.

\footnotetext{
$\dagger$ Aerospace Engineer, Intelligent Control and Autonomy Branch, Amy.K.Chicatelli@nasa.gov, AIAA Senior Member.

* Aerospace Engineer, Intelligent Control and Autonomy Branch, Aidan.W.Rinehart@nasa.gov, AIAA Member.

$\S$ Aerospace Engineer, Intelligent Control and Autonomy Branch, Thomas.S.Sowers@ nasa.gov.

*** Research Engineer, Intelligent Control and Autonomy Branch, Donald.L.Simon@ nasa.gov.
}

American Institute of Aeronautics and Astronautics 


\section{Motivation}

\section{A. Significance}

As previously noted, asymmetric thrust is cited as the cause of several Loss of Control aviation incidents and accidents. The sequence of events that lead to a PSM+ICR event can be described as follows. In a typical scenario, the aircraft's autopilot is being used by the crew and the autothrottle control is engaged. Due to a failure, an unintended asymmetric thrust condition occurs while the autopilot and autothrottle are engaged. Due to the automated flight control systems, the asymmetric thrust condition is initially managed, but it progressively increases because the flight controls reach their limits of effective control. When the autopilot disengages and the crew takes over control, there may not be enough control authority for the crew to sufficiently control the aircraft. In addition, the crew's response may be inappropriate and exacerbate the situation.

If the flight crew is aware of the developing thrust asymmetry, they should be able to identify the cause and take preventive or corrective action. Therefore, the detection of the asymmetric thrust condition is needed, and the annunciation of asymmetric thrust may be beneficial in warning the flight crew. However, as indicated in the Federal Aviation Administration (FAA) report ${ }^{4}$ for indications of propulsion system malfunctions, there are risks associated with thrust asymmetry annunciation and the only time when annunciation may be practical is when the autopilot is engaged. Many of the reported incidents and accidents of thrust asymmetry by themselves were not so significant that they were not recoverable. In addition, aircraft are required to be controllable in extreme asymmetric thrust conditions throughout typical flight profiles. For the purposes of this effort, the asymmetric thrust condition that leads to aircraft upset and to loss of control is of interest. Typically that condition occurs due to the lack of reaction or from the inappropriate action of the flight crew. It is in this way that an asymmetric thrust condition can become unmanageable.

\section{B. Improvement over State of the Art}

The flight crew is responsible for recognizing and responding to unintended asymmetric thrust conditions. Today, this is done by the pilots monitoring available engine cockpit instrumentation such as rotor speeds or engine pressure ratio, which provide an indication of engine power. If an imbalance in the power produced by the aircraft's engines is observed, the pilots must take appropriate action to address the issue. It is possible, when the autopilot is being used, the thrust imbalance can actually be exacerbated. If the flight control system is continually correcting for thrust asymmetry, the limits of control authority could be approached. In this case, recovery by the flight crew will be challenged by the suddenness of the event and the reduced margin for corrective action. Depending on its design, the autopilot will disengage at some point as it becomes unable to maintain the desired input flight conditions (i.e., heading, airspeed, altitude). When the autopilot disengages, the flight crew is given control of an aircraft that may have developed an unusual flight attitude with controls at or near their maximum deflection from the autopilot. In these situations, recovery depends on the proper response and skill of the flight crew.

As noted above, asymmetric thrust conditions can be masked from the flight crew when the automatic controls are being used. In order to add an alert for asymmetric thrust conditions, the thrust imbalance must be detected and confirmed when these controls are being used. The results of the research presented in this paper, will show that the detection and confirmation of asymmetric thrust conditions is possible. The simulation studies and pilot-in-the-loop tests that were conducted under this effort will show that reliable automated real-time detection of asymmetric thrust conditions is feasible.

\section{Asymmetric Thrust Detection and Confirmation}

For this research effort, three asymmetric thrust detection approaches are developed and evaluated. The first two are based on producing an estimate of the engine thrust for each engine and comparing those values with each other in order to determine a mismatch in the thrust. The first two approaches include a Kalman filter-based thrust estimation approach and a two-dimensional table lookup thrust estimation approach. The third method takes a fundamentally different approach. Instead of a monitoring for a mismatch in estimated thrust between engines it monitors for a mismatch between the commanded and actual power produced by an individual engine. This is done by monitoring the primary engine control parameter (typically either corrected fan speed or engine pressure ratio (EPR)), which is a proxy for the amount of thrust produced by the engine. If an engine is detected to be producing more/less thrust than commanded, that serves as confirmation that an asymmetric thrust condition is likely. The three detection approaches are described in the following sections.

American Institute of Aeronautics and Astronautics 


\section{A. Kalman Filter Approach}

A Kalman filter (KF) is an optimal linear estimator designed to estimate the unknown states of a dynamic system. It incorporates a dynamic model of the system and is designed to recursively update estimates by processing acquired system measurement data. Accounting for measurement noise and model uncertainty, the Kalman filter is designed to minimize the mean squared error in the estimated parameters. It is well suited for aircraft engine applications and several previous efforts have reported on the application of Kalman filters for onboard real-time aircraft engine performance estimation. ${ }^{5,6}$ In this study, an asymmetric thrust detection strategy based on Kalman filter estimation technology is considered. Here, a Kalman filter is designed and applied for each engine installed on the aircraft. To account for the nonlinear behavior inherent in an aircraft gas turbine engine, a piecewise linear Kalman filter design is applied. Individual linear Kalman filters are designed spanning the entire engine operating envelope and then combined and scheduled applying interpolation to account for changes in engine operating condition. In addition to estimating the dynamic states of the engine, the Kalman filter is also constructed to estimate states reflective of turbomachinery performance deterioration. ${ }^{7,8}$ In this fashion, that Kalman filter is able to account for deterioration induced performance changes in the engine. Additional details on the Kalman filter formulation and implementation for asymmetric thrust detection are provided in the subsections below.

\section{Kalman Filter Formulation}

The nonlinear model of an aircraft engine can be represented by the following equations

$$
\begin{aligned}
\dot{x} & =f(x, u, h) \\
y & =g(x, u, h) \\
z & =g_{z}(x, u, h)
\end{aligned}
$$

where $x$ and $u$ represent the vectors of engine state variables and control command inputs, respectively. The vector $h$ represents health parameters, such as efficiency or flow capacity, reflective of performance deterioration within the major modules of the engine. For given input values, the nonlinear functions $f, g$, and $g_{z}$ generate the vectors of state derivatives $\dot{x}$, sensed engine outputs $y$, and unmeasured engine outputs such as net thrust denoted by $z$. By linearizing the engine model at a given operating point, the following state-space equations are obtained:

$$
\begin{aligned}
\dot{x} & =A \underbrace{\left(x-x_{\text {trim }}\right)}_{\Delta x}+B \underbrace{\left(u-u_{\text {trim }}\right)}_{\Delta u}+L \underbrace{\left(h-h_{\text {ref }}\right)}_{\Delta h} \\
\dot{x} & =A \Delta x+B \Delta u+L \Delta h \\
\underbrace{y-y_{\text {trim }}}_{\Delta y} & =C \underbrace{\left(x-x_{\text {trim }}\right)}_{\Delta x}+D \underbrace{(\underbrace{\left.u-u_{\text {trim }}\right)}_{u})}_{\Delta u}+M \underbrace{\left(h-h_{\text {ref }}\right)}_{\Delta h} \\
\Delta y & =C \Delta x+D \Delta u+M \Delta h \\
\underbrace{z-z_{\text {trim }}}_{\Delta z} & =F \underbrace{\left(x-x_{\text {trim }}\right)}_{\Delta x}+G \underbrace{\left(u-u_{\text {trim }}\right)}_{\Delta u}+N \underbrace{\left(h-h_{r e f}\right)}_{\Delta h} \\
\Delta z & =F \Delta x+G \Delta u+N \Delta h
\end{aligned}
$$

Here, $A, B, C, D, F, G, L, M$, and $N$ are the state-space matrices reflecting system dynamics. The trim vectors, denoted by the subscript "trim," reflect the values of the state variables, commands, and measured and unmeasured outputs when the model is at steady-state (i.e., $\dot{x}=0$ ) at the given operating point. The vector $h_{\text {ref }}$ represents a reference health condition specified by the system designer. In Equation (2), parameter deviations relative to trim or reference conditions are denoted by the delta symbol $(\Delta)$. Through algebraic manipulation, Equation (2) can be rewritten to shift the health parameters to become state variables as shown in Equation (3):

American Institute of Aeronautics and Astronautics 


$$
\begin{aligned}
& {\left[\begin{array}{l}
\dot{x} \\
\dot{h}
\end{array}\right]=\left[\begin{array}{ll}
A & L \\
0 & 0
\end{array}\right]\left[\begin{array}{c}
\Delta x \\
\Delta h
\end{array}\right]+B \Delta u} \\
& \Delta y=\left[\begin{array}{ll}
C & M
\end{array}\right]\left[\begin{array}{l}
\Delta x \\
\Delta h
\end{array}\right]+D \Delta u \\
& \Delta z=\left[\begin{array}{ll}
F & N
\end{array}\right]\left[\begin{array}{l}
\Delta x \\
\Delta h
\end{array}\right]+G \Delta u
\end{aligned}
$$

Since engine performance deterioration evolves slowly in time, the health parameter states in Equation 3 are modeled without dynamics. Once the health parameters are augmented with the state variables, they can be estimated by applying a Kalman filter as long as the system is observable. However, a necessary condition for observability given the Equation 3 formulation is that there are at least as many measurements as health parameters. ${ }^{9}$ To construct a reduced-order state space system of appropriate dimension to enable Kalman filter formulation, consider a transformation matrix, $V^{*}$, that maps the health parameter vector, $h$, to a tuning vector of lower dimension, $q$, such that:

$$
q=V^{*} h
$$

An approximation for $h$ based on $q$ can be calculated using the pseudo inverse of $V^{*}$ :

$$
\hat{h}=V^{*+} \hat{q}
$$

Then, substituting Equation (5) into Equation (3) produces the following reduced-order state space system:

$$
\begin{aligned}
& \underbrace{\left[\begin{array}{c}
\dot{x} \\
\dot{q}
\end{array}\right]}_{\dot{x}_{x q}}=\underbrace{\left[\begin{array}{cc}
A & L V^{*+} \\
0 & 0
\end{array}\right]}_{A_{x q}} \underbrace{\left[\begin{array}{c}
\Delta x \\
\Delta q
\end{array}\right]}_{x_{x q}}+\underbrace{\left[\begin{array}{c}
B \\
0
\end{array}\right]}_{B_{x q}} \Delta u
\end{aligned}
$$

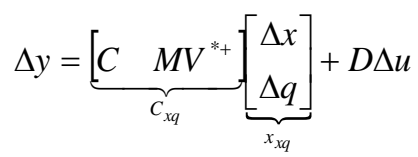

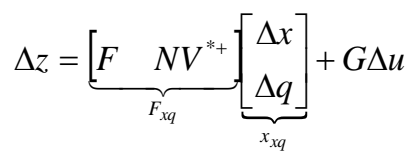

The choice of the transformation matrix is a design decision made prior to constructing the Kalman gains. In this study a technique, referred to as "optimal tuner selection," is employed to produce a transformation matrix that is a linear combination of all health parameters, and constructed such that the mean squared estimation error in the parameters of interest are minimized. ${ }^{7}$ In this case, the transformation matrix was selected to minimize the estimation error in net thrust. Given Equation (6), a linear Kalman filter at a given operating point can be formulated as:

$$
\begin{aligned}
& \dot{\hat{x}}_{x q}=\left(A_{x q}-K C_{x q}\right) \Delta \hat{x}_{x q}+B_{x q} \Delta u+K \Delta y \\
& \Delta \hat{y}=C_{x q} \Delta \hat{x}_{x q}+D \Delta u \\
& \Delta \hat{z}=F_{x q} \Delta \hat{x}_{x q}+G \Delta u
\end{aligned}
$$

American Institute of Aeronautics and Astronautics 
After individual linear Kalman filters are designed spanning the entire engine operating envelope, they are combined and scheduled applying interpolation to form the piecewise linear Kalman filter.

\section{Kalman Filter Implementation for Asymmetric Thrust Detection}

A block diagram of the piecewise linear Kalman filter implementation to estimate the thrust produced by an individual engine is shown in Figure 1. The Kalman filter requires engine sensed measurements $(y)$ and actuator inputs $(u)$. Parameter correction is applied to improve the interpolation between grid points in the piecewise linear Kalman filter. Trim and matrix information corresponding to the current operating point are retrieved applying a threedimensional interpolation scheme using altitude, Mach, and corrected fan speed as the scheduling parameters. The Kalman filter produces estimated corrected output deltas from trim consisting of state variables $\left(\Delta \hat{x}_{x q}\right)$, sensed measurements $\left(\Delta \hat{y}_{c}\right)$, and net thrust $\left(\Delta \hat{z}_{c}\right)$. Corrected net thrust is produced by summing the estimated delta in net thrust, $\Delta \hat{z}_{c}$, and net thrust at the trim condition, $z_{\text {trim. }}$.

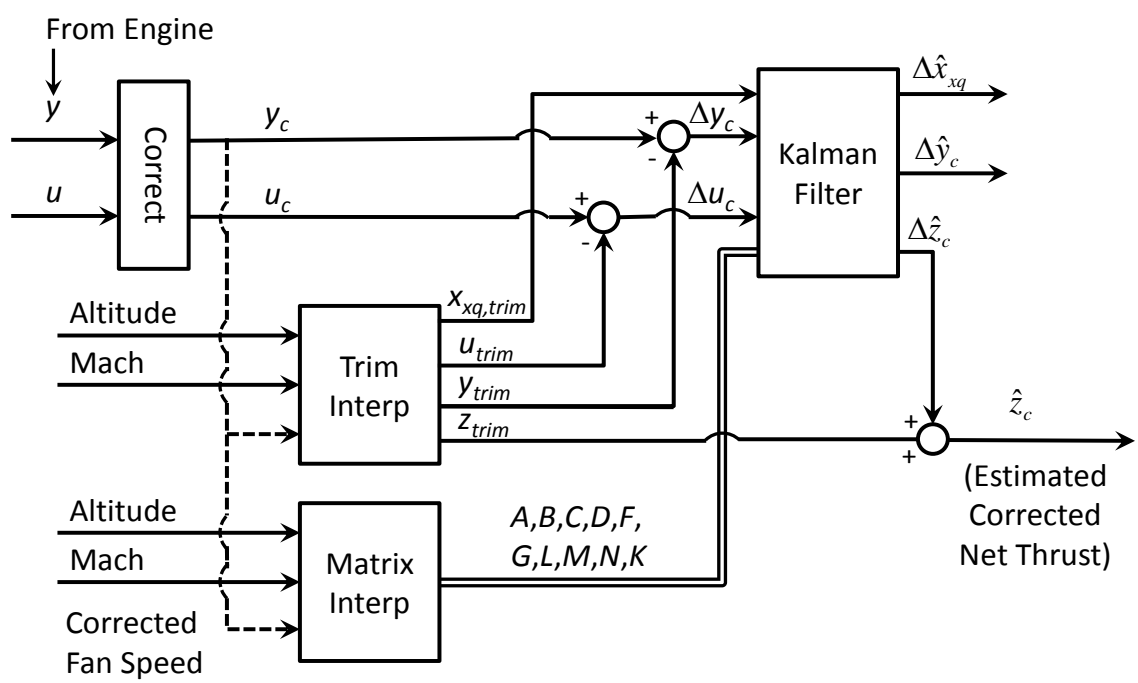

Figure 1. Kalman Filter-based Thrust Estimation

The Kalman filter estimated net thrust values for each engine are compared following the asymmetric thrust detection and confirmation logic as shown in Figure 2. The absolute difference in estimated corrected net thrust between the two engines is calculated and then converted to an absolute percent of maximum thrust. This absolute percent error signal is then compared to a pre-established detection threshold. When an exceedance of this threshold is detected and then persists for an established time duration, the asymmetric thrust condition is confirmed and annunciated.

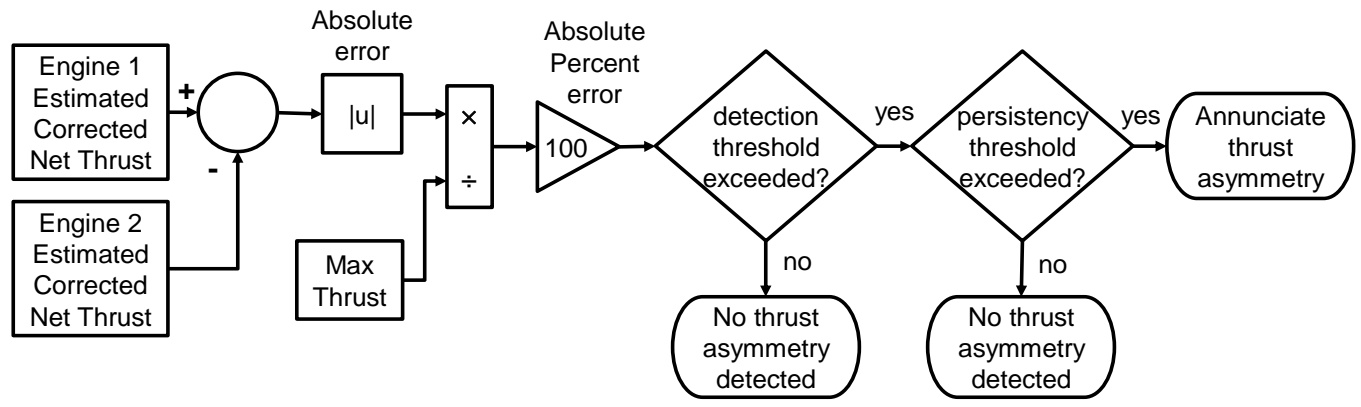

Figure 2. Asymmetric Thrust Detection and Confirmation Logic

Relative to the other asymmetric thrust detection approaches considered in this study, the primary benefit offered by the Kalman filter is the estimation accuracy it enables. It is designed to account for transient engine behavior and

American Institute of Aeronautics and Astronautics 
turbomachinery deterioration induced changes in engine performance. However, it is a relatively complex solution in terms of processing requirements compared to the other approaches described below.

\section{B. Table Lookup Approach}

This asymmetric thrust detection approach estimates the net thrust of each engine applying a two-dimensional table lookup approach based on corrected fan speed and Mach number as shown in Figure 3. The lookup table data is created from steady state data generated using an engine model reflecting mid-life (i.e., $50 \%$ deteriorated) engine performance. The model is run at operating points over the entire flight envelope of the engine spanning a range of corrected fan speeds, Mach number, and altitude settings. Then, for each corrected fan speed and Mach number combination, corrected net thrust results generated over the range of altitudes considered are averaged to produce the two-dimensional lookup model.

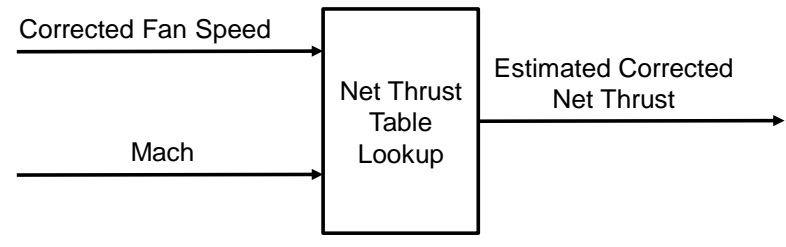

Figure 3. Table Lookup Thrust Estimation Approach

An estimated corrected net thrust value is produced for each engine, then these estimated thrust values are processed applying the same asymmetric thrust detection and confirmation system as shown previously in Figure 2. As compared to the Kalman filter approach, the table lookup method is simpler in design, which is a benefit for a flight software application. However, unlike the Kalman filter, the table lookup method does not account for engine performance deterioration or engine transient dynamics.

\section{Sensed and Commanded Power Comparison Approach}

The sensed and commanded power comparison approach is fundamentally different from the previously described Kalman filter and table lookup approaches. Instead of estimating and comparing the thrust between engines, this approach monitors for a mismatch between commanded and delivered power in an individual engine. It does so by monitoring the engine's control parameter, corrected fan speed or EPR for most turbofan engines, which serves as a proxy for the actual thrust produced by the engine. A block diagram representation of the approach is shown in Figure 4. Sensed power (i.e., corrected fan speed or EPR) is directly read from available engine measurements. Commanded power is based on the engine power setpoint control logic, which is a function of operating conditions (altitude, Mach, ambient temperature) and throttle power lever angle (PLA) setting. The logic compares sensed and commanded power setting, and if a mismatch of sufficient magnitude and persistency is found, a power mismatch condition is annunciated. A benefit of this approach is that it guards against thrust asymmetry alerts due to pilot-intentional split throttle command scenarios. The goal of this research is to identify methods capable of detecting unintended asymmetrical thrust conditions. The two thrust estimation methods discussed require additional logic to suppress alerts when the asymmetric thrust is intended. Additionally, the EPR comparison approach is able to provide an indication of which engine is the culprit for producing the asymmetric thrust condition-generally there should never be a mismatch between commanded and delivered EPR during nominal conditions. Conversely, the previously described Kalman filter and table lookup approach are non-specific as they simply indicate that a thrust asymmetry exists. They do not aid in identifying which engine is malfunctioning. Like the previously described table lookup approach, the logic is relatively simple. However, unlike the Kalman filter approach, it does not account for engine performance deterioration.

American Institute of Aeronautics and Astronautics 


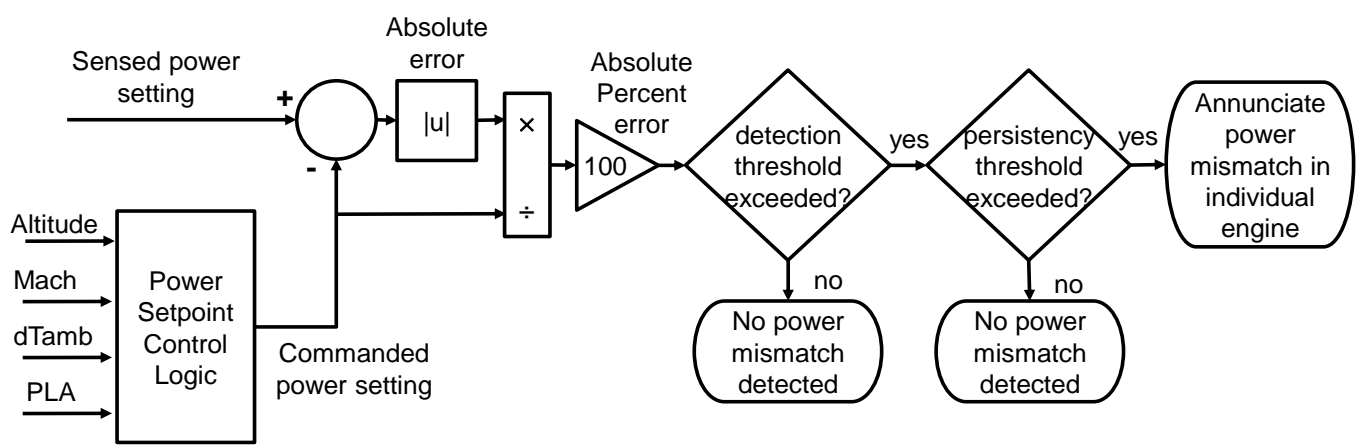

Figure 4. Sensed and Commanded Power Comparison Approach

\section{Application and Results}

This section will present simulation results illustrating the application and comparison of the three asymmetric thrust detection approaches. First, results from a Monte Carlo simulation study conducted to assess the detection sensitivity of the three approaches is presented. Then, results from the application of the approaches in a pilot-in-the loop flight simulation facility are presented.

\section{A. Asymmetric Thrust Detection Sensitivity Study}

In order to evaluate the detection sensitivity of the three asymmetric thrust approaches, a simulation study is conducted to quantify the average minimum thrust asymmetry that can be detected by each approach in the presence of representative system uncertainty. This study is conducted using the NASA-developed Commercial Modular AeroPropulsion System Simulation 40k (C-MAPSS40k), a high bypass turbofan engine model in the 40,000 pound thrust category. ${ }^{10}$ C-MAPSS40k is a generic turbofan engine model intended for controls and diagnostics research and development purposes. It includes a closed-loop controller with EPR as the primary engine control parameter. C-MAPSS40k sensor measurement, actuators, and input parameters are shown in Table 1. C-MAPSS40k input parameters allow the engine to be operated over a range of operating conditions and power settings. Additional inputs allow the end user to specify the engine's level of performance deterioration (ranging from a new engine to a fully degraded engine) and to enable the inclusion of representative sensor measurement noise in the engine outputs. Representative variations in these inputs are included in the sensitivity study to assess the accuracy of each of the three methods. Utilizing the C-MAPSS40k model, a piecewise linear Kalman filter and a two-dimensional table lookup model are constructed to estimate thrust over the entire engine operating envelope. The sensed and commanded power comparison approach is designed to compare sensed and commanded EPR. This is performed by comparing sensed EPR (the ratio of P5 to P2) to commanded EPR as specified by the C-MAPSS40k control logic.

Table 1. C-MAPSS40k sensor measurements, actuators, and input parameters

\begin{tabular}{|c|c|c|c|c|c|}
\hline \multicolumn{2}{|c|}{ Sensor Measurements } & \multicolumn{2}{|c|}{ Actuators } & \multicolumn{2}{|l|}{ Inputs } \\
\hline $\mathrm{Nf}$ & Fan speed & Wf & Fuel flow & Alt & Altitude \\
\hline $\mathrm{Nc}$ & Core speed & VSV & Variable stator vane & MN & Mach number \\
\hline $\mathrm{P} 2$ & Inlet total pressure & VBV & Variable bleed valve & PLA & Power lever angle \\
\hline $\mathrm{T} 2$ & Inlet total temperature & & & dTamb & Ambient temperature deviation \\
\hline $\mathrm{P} 25$ & HPC inlet total pressure & & & & relative to standard day conditions \\
\hline $\mathrm{T} 25$ & HPC inlet total temperature & & & Det & Performance deterioration level \\
\hline & HPC exit static pressure & & & Noise & Measurement noise enabled or \\
\hline T3 & HPT exit total temperature & & & & disabled (discrete input) \\
\hline
\end{tabular}

\begin{tabular}{|ll|}
\hline P5 & LPT exit total pressure \\
\hline T5 & LPT exit total temperature \\
\hline
\end{tabular}

Given the designs of the three asymmetry detection approaches, a Monte Carlo simulation study is conducted to assess and compare the sensitivity of the approaches. For the purposes of conducting this study, two C-MAPSS40k engine simulations are run in parallel to represent a twin engine aircraft. Each Monte Carlo trial is defined to be 10

American Institute of Aeronautics and Astronautics 
minutes in duration and applies pseudo-randomly assigned simulation inputs of engine operating condition, deterioration level, and sensor measurement noise. Here, actual flight operating condition profiles are obtained from a commercial aircraft flight operation quality assurance (FOQA) data set. Quasi steady-state cruise segments 10 minutes in duration are identified and extracted from the FOQA data set, and the corresponding time histories of altitude, Mach, and PLA serve as inputs into the C-MAPSS40k simulations. A total of 216 steady-state cruise segments were identified within the FOQA data set. Additionally, for each 10 minute trial the deterioration level of each engine is randomly assigned through the C-MAPSS40k deterioration parameter and sensor noise is enabled.

To enable a common basis of comparison between the three asymmetric thrust detection approaches, detection logic for each approach was defined to produce a common false alarm rate. All three methods applied a threshold exceedance persistency check of 6.5 seconds in duration. The corresponding detection threshold was manually adjusted until two of the 216 steady-state cruise segments experienced a false alarm. For these cases, no thrust asymmetry existed beyond that caused by the different level of performance deterioration assigned to each engine and noise in the sensor measurements used by the closed-loop control system, which produce a relatively small mismatch in thrust. The thresholds applied for the three detection approaches are shown in Table 2. It should be emphasized that the detection thresholds applied for this study are much smaller than what would be reasonable to implement in practice as a false alarm rate of $0.93 \%$ ( 2 of 216 ) vastly exceeds what would be considered acceptable. However, establishment of a common false alarm rate does provide a common basis in order to enable comparison of the methods.

Table 2. Applied detection thresholds for the three asymmetric thrust detection approaches

\begin{tabular}{|l|c|c|c|}
\hline Method & Threshold & Persistency & False Alarms \\
\hline \hline Kalman filter & $0.187 \%$ & $6.5 \mathrm{sec}$ & 2 of 216 trials \\
\hline Table lookup & $0.087 \%$ & $6.5 \mathrm{sec}$ & 2 of 216 trials \\
\hline Sensed and commanded EPR comparison & $0.95 \%$ & $6.5 \mathrm{sec}$ & 2 of 216 trials \\
\hline
\end{tabular}

Given the defined detection thresholds for each method, the approaches were then evaluated and compared to determine their sensitivity in detecting asymmetric thrust events. For this assessment, two C-MAPSS40k simulations were once again run in parallel to produce 10 minute time histories for a twin engine aircraft. As before, sensor measurement noise was enabled and inputs consisting of FOQA operating condition data and randomly assigned deterioration levels were applied. To simulate a thrust mismatch between the engines, engine \#1 was setup to experience an uncommanded slow linear bias increase in PLA above its actual true PLA setting. This resulted in an overthrust condition for engine \#1, where it produced more thrust than commanded. A Monte Carlo simulation study was conducted, and the average absolute percent thrust mismatch between the engines at the time of detection was quantified. This average is based on results from the same 216 steady-state cruise cases used to establish the detection thresholds. The results are summarized in Table 3 and show the average and standard deviation of the absolute percent corrected thrust asymmetry at the time of detection. It is observed that the table lookup approach is able to detect the smallest levels of thrust asymmetry, followed by the Kalman filter approach, and then the EPR comparison approach. The table lookup approach relies on corrected fan speed to estimate thrust, because they are highly correlated variables. For C-MAPSS40k, the relationship between corrected fan speed and thrust is not significantly impacted by engine performance deterioration. Additionally, the corrected fan speed measurement noise is relatively small. As such, the table lookup method performs well in estimating and comparing engine produced thrust at the quasi-steady-state cruise conditions considered. The Kalman filter provides comparable performance to the table lookup model. The slight increase in average detectable thrust asymmetry is attributed to the sensor noise, which is more prominent in the additional C-MAPSS40k sensors used in the Kalman filter implementation. The sensed and commanded EPR comparison approach utilizes P2 and P5 sensor measurements to calculate sensed EPR. These sensors exhibit more noise than the fan speed sensor does. Additionally, there is a lag between commanded and delivered EPR which comes into play due to oscillations in the PLA command and operating conditions within the FOQA data. As such, the sensed vs. commanded EPR comparison approach exhibits a larger magnitude and standard deviation in its average minimum detectable thrust asymmetry. 
Table 3. Average minimum level of detectable thrust asymmetry

\begin{tabular}{|l|c|c|}
\hline Method & $\begin{array}{c}\text { Average absolute percent of } \\
\text { corrected thrust asymmetry at the } \\
\text { time of detection }\end{array}$ & Standard Deviation \\
\hline \hline Kalman filter & $0.9664 \%$ & $2.7792 \%$ \\
\hline Table lookup & $0.7647 \%$ & $2.1976 \%$ \\
\hline Sensed and commanded EPR comparison & $2.7672 \%$ & $4.0936 \%$ \\
\hline
\end{tabular}

\section{B. Piloted Flight Simulation Evaluation}

In order to further assess the capabilities of the asymmetric thrust detection approaches, a pilot-in-the-loop flight simulation evaluation is conducted where simulated failure scenarios causing asymmetric thrust conditions are introduced. This evaluation allow the techniques to be evaluated under more realistic operating conditions including transient operating conditions and pilot interactions with the aircraft. The subsections below will describe the test setup and example results of asymmetric thrust detection in the flight simulator environment.

\section{NASA GRC Flight Simulation Testbed}

The NASA GRC flight simulation testbed is capable of real-time piloted evaluation of the asymmetric thrust detection methods and provides a unique opportunity for this purpose. This demonstration vehicle generally meets FAA guidelines; so, it provides a valid test environment. ${ }^{11}$ In this study, the flight simulation testbed utilized the twoengine transport class Model (TCM) aircraft simulation developed by NASA LaRC ${ }^{12}$ and the C-MAPPS40k model developed by NASA GRC for each engine. The integration of the TCM aircraft simulation and the C-MAPSS40k engine simulation is referred to as the TCM with enhanced engine control (EEC). The TCM EEC has been used for NASA GRC aero-propulsion projects, and it serves as a realistic demonstration vehicle. Shown in Figure 5 is a picture of the cockpit from the flight simulation testbed.

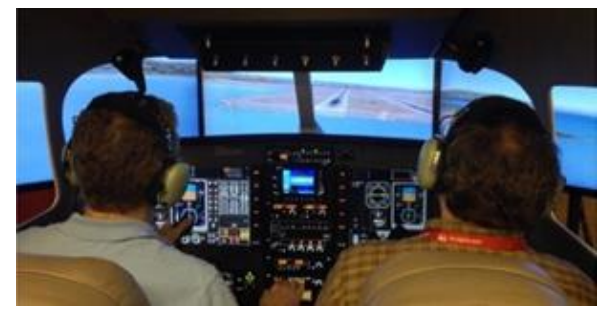

Figure 5 NASA GRC Flight Simulation Testbed

\section{Integration of the Asymmetric Thrust Failure Scenarios and Detection Methods}

Modifications to the TCM EEC flight simulation were completed for including the asymmetric thrust failure scenarios, which included stuck throttle failures as well as EPR-type failures. For the stuck throttle failures, the ability to fail either throttle was implemented in the two-engine TCM EEC simulation. From the reported incidents and accidents, the typical asymmetric thrust failure scenario usually involves one engine that is not responsive to throttle movement. The EPR failure scenarios were implemented as failures in the EPR sensor or in the fuel meter valve (FMV) system.

The three asymmetric thrust detection methods were integrated into the TCM EEC for subsequent evaluation. The two detection methods based on comparing estimated thrust, the Kalman filter and table lookup approaches, were implemented as previously shown in Figure 2 and the EPR comparison approach was implemented as shown in Figure 4. For asymmetric thrust detection and confirmation all three methods applied a persistency check of 6.5 seconds. The estimated thrust mismatch threshold for the Kalman filter and the table lookup model was set to ten percent, and the EPR mismatch threshold was set to five percent. The ten percent estimated thrust mismatch threshold was selected to be consistent with current engine failure indication logic activation levels ${ }^{4}$. The ten percent thrust mismatch was selected as a practical threshold already implemented in the industry, whereas the thrust mismatch values identified in section IV provided a common ground for comparison between methods. The threshold values obtained in section 
IV are not practical for implementation in real world settings due to the considerable high false positive rate. The five percent threshold applied for the EPR comparison approach was selected to provide the capability to detect comparable thrust imbalance levels of approximately $10 \%$.

\section{Examples Asymmetric Thrust Failures}

From previous pilot-in-the-loop testing and the available accident reports and documentation, the stuck throttle failure provides realistic emergency conditions for the pilot to handle; so, it was used for this preliminary evaluation of the asymmetric thrust detection methods. The modeled stuck throttle failures were evaluated in the GRC flight simulation testbed. These pilot-in-the-loop tests were used to demonstrate the capabilities of the stuck throttle subsystems that were implemented in the TCM EEC. The failure where the throttle is stuck at its last position at a user-defined time was selected as the failure scenario. For this specific example, the pilot has finished the cruise portion of the flight and is starting to make the approach to land. Without the pilot's knowledge, the stuck throttle failure was inserted into the real-time flight simulation. The pilot recognized that there was a throttle failure, but a comparison between the pilot's reaction time and when the asymmetric thrust condition was detected was not evaluated. Since, the primary purpose for this demonstration was to show the integration of the failure scenario and asymmetric thrust detection methods with the real-time flight simulation.

Figure 6 shows an example of the correct detection and annunciation of an asymmetric thrust event based on the Kalman filter thrust estimation approach. The top plot shows the estimated net thrust for engine one and engine two. At time equal to 15 seconds, engine one experiences a stuck throttle failure. The middle plot shows the absolute net thrust difference between engines one and two along with the ten percent target detection threshold. The bottom plot shows the asymmetric thrust detection logic output. Asymmetric thrust is correctly detected and confirmed after the event persists for greater than 6.5 seconds. The other approaches were also found to be able to successfully detect simulated asymmetric thrust scenarios. An analysis similar to the one that was performed for the sensitivity study was not repeated again due to the many pilot-in-the-loop simulations that would have to be performed. Although, more than one pilot-in-the-loop test was performed, the results from those tests are similar to the one presented here.
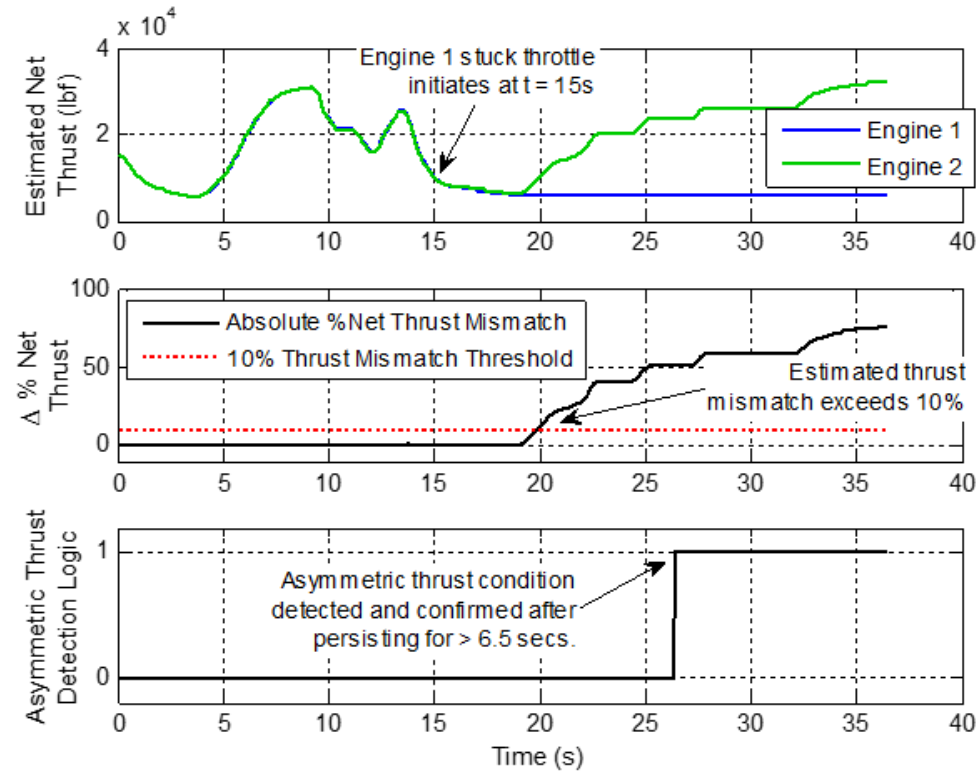

Figure 6. Detection and Confirmation of Asymmetric Thrust Based on the Kalman Filter Thrust Estimation Approach

\section{Discussion}

The three asymmetric thrust detection methods presented in this paper all performed as expected in detecting thrust asymmetry between engines of $10 \%$ or more, as demonstrated in a pilot-in-the-loop test. As such, all are potential candidates for detecting large asymmetric thrust events of the magnitude that might pose a safety concern. The two methods that monitored for a mismatch in the estimated thrust produced between engines (i.e., the Kalman filter and table lookup approach) were both found to be effective in detecting the simulated asymmetric thrust events. However, the Kalman filter is more complex, and was not found to yield a significant detection benefit over the simpler table lookup approach when applied to C-MAPSS40k. Additionally, some practical implementation challenges associated 
with the two thrust comparison methods are acknowledged. First, they can produce alarms in the event that an intentional split throttle setting exists. Additional logic would have to be added for the methods to suppress alerts when intentional asymmetric thrust is commanded. For example, the detection logic could be designed to only be engaged when the automatic controls are activated and disengaged during all other times. Another limitation of the estimated thrust comparison approaches is that the detection of a thrust asymmetry is nonspecific and does not aid the crew in determining which engine is malfunctioning or why the engine is malfunctioning. ${ }^{4}$ Additional diagnostic logic, or manual troubleshooting would be necessary to determine the problem and the appropriate mitigating actions necessary to address the thrust asymmetry. The sensed and commanded power setting comparison approach was also found to be an effective and simple design. Furthermore, it is able to identify the specific engine that is the culprit for producing either overthrust or a thrust shortfall. One issue with the sensed vs. commanded approach is that it is limited to detecting only those events that result in a mismatch between commanded and sensed power output. If there is an issue upstream such as a throttle linkage issue resulting in the wrong PLA command being provided to the detection logic, this approach will not be able to detect such a scenario. For any of the three detection methods considered, additional development and maturation would be necessary to enable practical implementation. For additional information on recommendations and challenges associated with providing aircraft indications of thrust asymmetry and other propulsion malfunctions, readers are referred to Ref. 4.

\section{Summary and Conclusions}

The flight crew is responsible for recognizing and responding to unintended asymmetric thrust conditions. However, asymmetric thrust is often not recognized because of an increasing reliance on automated flight controls (i.e. autopilot). Therefore, an asymmetric thrust condition can be masked from the flight crew until a flight control limit is reached when recovery is difficult or not possible. Simulation studies conducted under this effort have shown that reliable automated real-time detection of asymmetric thrust conditions is feasible. Three asymmetric thrust detection approaches were developed and evaluated including two approaches designed to estimate and compare the thrust between engines (a Kalman filter thrust estimation technique and a corrected fan speed table lookup model) and a third approach designed to detect a mismatch between commanded and delivered Engine Pressure Ratio (EPR) in an individual engine. The approaches were evaluated in the NASA GRC flight simulation testbed under a variety of failure scenarios. All approaches were found to be successful in detecting asymmetric conditions. In particular, for the stuck throttle failure, the asymmetric thrust detection and confirmation was shown to be successful.

\section{References}

1 "Propulsion System Malfunction Plus Inappropriate Crew Response (PSM+ICR), Aerospace Industries Association and the European Association of Aerospace Industries Project Report, Vol. 1, November 1 (1998).

2 "Propulsion System Malfunction Plus Inappropriate Crew Response (PSM+ICR) Human Factors Report," Flight Safety Digest November-December (1999).

3 “Turbofan Engine Malfunction Recognition and Response," FAA Engine and Propeller Directorate Final Report, July 17 (2009).

4 "Indications of Propulsion System Malfunctions," Final Report, DOT/FAA/AR-03/72, November (2004).

${ }^{5}$ Luppold, R. H. Roman, J. R., Gallops, G. W., Kerr, L. J., "Estimating In-Flight Engine Performance Variations Using Kalman Filter Concepts," AIAA-89-2584, AIAA 25th Joint Propulsion Conference, Monterey, CA, July 10-12, (1989).

6 Volponi, A., "Enhanced Self-Tuning On-Board Real-Time Model (eSTORM) for Aircraft Engine Performance Health Tracking," NASA CR-2008-215272, July (2008).

${ }^{7}$ Simon, D. L., and Garg, S., "Optimal Tuner Selection for Kalman Filter-Based Aircraft Engine Performance Estimation," Journal of Engineering for Gas Turbines and Power, Vol. 132 / 031601-1-10 (2010).

${ }^{8}$ Simon, D. L., Armstrong, J. B., Garg, S., “Application of an Optimal Tuner Selection Approach for On-Board Self-Tuning Engine Models," Journal of Engineering for Gas Turbines and Power, Vol. 134 / 04160-1-11 (2012).

${ }^{9}$ España, M. D., "Sensor Biases Effect on the Estimation Algorithm for Performance-Seeking Controllers," ASME Journal of Propulsion and Power, Vol. 10, pgs. 527-532 (1994).

${ }^{10}$ May, R. D., Csank, J., Litt, J., Guo, T., “Commercial Modular Aero-Propulsion System Simulation (C-MAPSS40k) User’s Guide," NASA TM 216831, September (2010).

${ }^{11}$ Litt, J., Sowers, S., Lieu, J., Owen, K., Guo, T., "Validation of an Integrated Airframe and Turbofan Engine Simulation for Evaluation of Propulsion Control Modes," AIAA SciTech, January (2015).

${ }^{12}$ Hueschen, R. M., "Development of the Transport Class Model (TCM) Aircraft Simulation From a Sub-Scale Generic Transport Model (GTM) Simulation,” NASA/TM-2011-217169, August (2011).

American Institute of Aeronautics and Astronautics 